\title{
ON THE EXPANSION OF THE REMAINDER IN THE NEWTON-COTES FORMULA*
}

\author{
BY \\ J. V. USPENSKY
}

1. In Newton's method for approximate evaluation of definite integrals the interval of integration, say $(0,1)$, is divided into a certain number $n$ of equal parts and the integral of a given function $f(x)$ is assumed to be approximately equal to the integral of the interpolation polynomial of degree $n$ which at the points of subdivision has the same values as $f(x)$. The resulting approximate formula

$$
\int_{0}^{1} f(x) d x=A_{0} f(0)+A_{1} f\left(\frac{1}{n}\right)+\cdots+A_{n} f(1)
$$

is known as the Newton-Cotes quadrature formula. The coefficients $A_{0}$, $A_{1}, \cdots, A_{n}$ depend on the number of divisions $n$ and their values have been computed by Cotes for $n \leqq 10$. In the following we shall call them "Cotes coefficients."

Formula (1) is exact for an arbitrary polynomial $f(x)$ of degree not exceeding $n$. However, since for an even $n$

$$
\int_{0}^{1} x\left(x-\frac{1}{n}\right)\left(x-\frac{2}{n}\right) \cdots(x-1) d x=0,
$$

formula (1) will be exact even for polynomials of degree $n+1$ if $n$ is even. Strange as it may seem, the expression for the remainder in the NewtonCotes formula was unknown till quite recently. It was only in 1922 and 1924 that J. F. Steffenson succeeded in giving a genuine expression of that remainder first for an even and then for an odd $n$. $\dagger$ In this paper we shall show that the remainder in the Newton-Cotes formula possesses an expansion in all respects quite similar to the classical Euler-Maclaurin expansion, which fact is interesting in itself and may be very useful in practice. The method by which this result is obtained is similar to that employed in our paper $O n$ an expansion of the remainder in the Gaussian quadrature formula, $\ddagger$ but besides

* Presented to the Society, September 7, 1934; received by the editors August 9, 1934.

$\dagger$ The detailed derivation of the remainder in the Newton-Cotes formula can be found in an excellent book by J. F. Steffenson, Interpolation, Baltimore, Williams and Wilkins, 1927.

$\ddagger$ Bulletin of the American Mathematical Society, vol. 40 (1934). 
simple means used in that paper it requires an elaborate study of certain properties of Cotes coefficients.*

2. In a paper Sur les valeurs asymptotiques des coefficients de Cotes $\dagger$ I have shown that for large $n$ and uniformly in $k(1 \leqq k \leqq n-1)$

$$
A_{k} \sim \frac{C_{n}^{k}}{n(\log n)^{2}}\left[\frac{(-1)^{k-1}}{k}+\frac{(-1)^{n-k-1}}{n-k}\right]
$$

while

$$
A_{0}=A_{n} \sim \frac{1}{n \log n} .
$$

These formulas show that for sufficiently large $n$

$$
(-1)^{k-1} A_{k}>0, A_{0}>0,
$$

if $1 \leqq k \leqq \frac{1}{2} n$; as to the signs of the remaining coefficients, they result from the elementary relation

$$
A_{n-k}=A_{k} .
$$

Now it is very important to determine the least value of $n$ for and after which inequalities (2) hold. It will be shown that $A_{0}>0$ always and that $(-1)^{k-1} A_{k}>0\left(1 \leqq k \leqq \frac{1}{2} n\right)$ for even $n \geqq 8$ and odd $n \geqq 11$.

3. From our article quoted above $\ddagger$ we take the following expression of $A_{k}(1 \leqq k \leqq n-1)$ :

$$
\begin{aligned}
A_{k}=\frac{1}{n} & +\frac{n+1}{n} C_{n}^{k}(-1)^{k-1}\left[\int_{0}^{1} \xi^{k}(1-\xi)^{n-k} d \xi \int_{-\infty}^{\log ((1-\xi) / \xi)} \frac{e^{k x}}{\pi^{2}+x^{2}} d x\right. \\
& \left.+(-1)^{n} \int_{0}^{1} \xi^{n-k}(1-\xi)^{k} d \xi \int_{-\infty}^{\log ((1-\xi) / \xi)} \frac{e^{(n-k) x}}{\pi^{2}+x^{2}} d x\right] .
\end{aligned}
$$

Introducing here instead of $x$ a new variable $t$ defined by

$$
\frac{\xi}{1-\xi} e^{x}=t
$$

we can present $A_{k}$ in the form

* I must express my thanks to Professor G. Pólya for some very helpful suggestions in connection with this investigation.

$\dagger$ Bulletin of the American Mathematical Society, vol. 31 (1925), pp. 145-156. See also G. P6lya, Ueber die Konvergenz von Quadraturverfahren, Mathematische Zeitschrift, vol. 37(1933).

$\ddagger$ Attention should be called to the fact that many formulas on p. 147 of that article are marred by typographical errors. 
(3)

$$
\begin{array}{r}
A_{k}=\frac{1}{n}+\frac{n+1}{n}(-1)^{k-1} C_{n}^{k} \int_{0}^{1} \int_{0}^{1} \frac{\phi(t)(1-\xi)^{n} d \xi d t}{\pi^{2}+\left(\log \frac{1-\xi}{\xi} t\right)^{2}} \\
\phi(t)=t^{k-1}+(-1)^{n} t^{n-k-1}
\end{array}
$$

whence

$$
(-1)^{k-1} n A_{k}=(-1)^{k-1}+(n+1) C_{n}^{k} \int_{0}^{1} \int_{0}^{1} \frac{\phi(t)(1-\xi)^{n} d \xi d t}{\pi^{2}+\left(\log \frac{1-\xi}{\xi} t\right)^{2}}
$$

and

$$
\begin{aligned}
(-1)^{k-1} n A_{k}>(-1)^{k-1}+(n+1) C_{n}^{k} & \int_{0}^{1 / 2}(1-\xi)^{n} F(\xi) d \xi, \\
F(\xi) & =\int_{\xi /(1-\xi)}^{1} \frac{t^{k-1}+(-1)^{n} t^{n-k-1}}{\pi^{2}+\left(\log \frac{1-\xi}{\xi} t\right)^{2}} d t .
\end{aligned}
$$

From this inequality it follows immediately that for an odd $k \leqq n / 2$ the coefficient $A_{k}$ is positive.

Integrating by parts we find

$$
\begin{aligned}
\int_{\xi /(1-\xi)}^{1} \frac{t^{k-1}+(-1)^{n} t^{n-k-1}}{\pi^{2}+\left(\log \frac{1-\xi}{\xi} t\right)^{2}} d t> & \frac{\frac{1}{k}+\frac{(-1)^{n}}{n-k}}{\pi^{2}+\left(\log \frac{1-\xi}{\xi}\right)^{2}} \\
& -\frac{1}{\pi^{2}}\left[\frac{1}{k}\left(\frac{\xi}{1-\xi}\right)^{k}+\frac{(-1)^{n}}{n-k}\left(\frac{\xi}{1-\xi}\right)^{n-k}\right],
\end{aligned}
$$

and hence

because

$$
\begin{aligned}
(n+1) C_{n}^{k} \int_{0}^{1 / 2}(1-\xi)^{n} d \xi \int_{\xi /(1-\xi)}^{1} \frac{t^{k-1}+(-1)^{n} t^{n-k-1}}{\pi^{2}+\left(\log \frac{1-\xi}{\xi} t\right)^{2}} d t \\
>(n+1) C_{n}^{k}\left(\frac{1}{k}+\frac{(-1)^{n}}{n-k}\right) \int_{0}^{1 / 2} \frac{(1-\xi)^{n} d \xi}{\pi^{2}+\left(\log \frac{1-\xi}{\xi}\right)^{2}}-\frac{1}{k \pi^{2}}
\end{aligned}
$$

$$
\begin{aligned}
\frac{(n+1) C_{n}^{k}}{k} \int_{0}^{1 / 2} \xi k(1-\xi)^{n-k} d \xi+(-1)^{n} \frac{(n+1) C_{n}^{k}}{n-k} \int_{1 / 2}^{1} \xi^{k}(1-\xi)^{n-k} d \xi & \\
& <\frac{(n+1) C_{n}^{k}}{k} \int_{0}^{1} \xi^{k}(1-\xi)^{n-k} d \xi=\frac{1}{k}
\end{aligned}
$$


Consequently for an even $k \leqq n / 2$

(4) $-n A_{k}>(n+1) C_{n}^{k}\left(\frac{1}{k}+\frac{(-1)^{n}}{n-k}\right) \int_{0}^{1 / 2} \frac{(1-\xi)^{n} d \xi}{\pi^{2}+\left(\log \frac{1-\xi}{\xi}\right)^{2}}-1-\frac{1}{k \pi^{2}}$.

4. In order to draw the desired conclusions from this inequality we must find a sufficiently precise and tractable lower limit of the integral

$$
K_{n}=\int_{0}^{1 / 2} \frac{(1-\xi)^{n}}{\pi^{2}+\left(\log \frac{1-\xi}{\xi}\right)^{2}} d \xi
$$

Consider a more general integral

$$
K_{n}(\alpha)=\int_{0}^{1 / 2} \frac{\xi^{-\alpha}(1-\xi)^{n+\alpha}}{\pi^{2}+\left(\log \frac{1-\xi}{\xi}\right)^{2}} d \xi, \quad-n<\alpha \leqq 0,
$$

which for $\alpha=0$ reduces to $K_{n}$. Since

$$
\frac{d^{2} K_{n}(\alpha)}{d \alpha^{2}}+\pi^{2} K_{n}(\alpha)=\int_{0}^{1 / 2}(1-x)^{n+\alpha} x^{-\alpha} d x=\phi(\alpha),
$$

we obtain, by integrating this equation,

$$
K_{n}(\alpha)=K_{n}(-2) \cos \pi \alpha+\frac{K_{n}^{\prime}(-2)}{\pi} \sin \pi \alpha+\frac{1}{\pi} \int_{-2}^{\alpha} \phi(x) \sin \pi(\alpha-x) d x .
$$

Taking here $\alpha=0$ we get

$$
K_{n}=K_{n}(-2)+\frac{1}{\pi} \int_{0}^{2} \phi(-x) \sin \pi x d x
$$

But

$$
\phi(-x)=\int_{0}^{1 / 2} \xi^{x}(1-\xi)^{n-x} d \xi=\frac{\Gamma(1+x) \Gamma(n+1-x)}{\Gamma(n+2)}-\frac{\vartheta}{2^{n} n}
$$

and $K_{n}(-2)>0$; consequently

$$
0<\vartheta<1 \text { if } n \geqq 5 \text {, }
$$

$$
K_{n}>\frac{1}{\pi} \int_{0}^{2} \omega(x) \sin \pi x d x-\frac{1}{2^{n-1} n \pi^{2}},
$$

where

$$
\omega(x)=\frac{\Gamma(1+x) \Gamma(n+1-x)}{\Gamma(n+2)} .
$$


As

$$
\omega(x)-\omega(x+1)=\frac{n-1-2 x}{n-x} \omega(x),
$$

we shall have a fortiori

$$
K_{n}>\frac{n-3}{(n-1) \pi} \int_{0}^{1} \omega(x) \sin \pi x d x-\frac{1}{2^{n-1} n \pi^{2}} .
$$

On the other hand $\Gamma(1+x)>e^{-C x}$, where $C$ is Euler's constant,

and

$$
\frac{\Gamma(n+1-x)}{\Gamma(n+1)}>e^{-x \log (n+1)}
$$

$$
\frac{1}{\pi} \int_{0}^{1} e^{-x[C+\log (n+1)]} \sin \pi x d x=\frac{1}{\pi^{2}+\{C+\log (n+1)\}^{2}}\left(1+\frac{e^{-C}}{n+1}\right) .
$$

Hence, finally,

$$
(n+1) K_{n}>\frac{n-3}{n-1} \frac{1}{\pi^{2}+\{C+\log (n+1)\}^{2}}\left(1+\frac{e^{-C}}{n+1}\right)-\frac{n+1}{2^{n-1} n \pi^{2}},
$$

and, taking into account inequality (4),

$$
\begin{aligned}
-n A_{k}> & C_{n}{ }^{k}\left(\frac{1}{k}+\frac{(-1)^{n}}{n-k}\right)\left[\frac{n-3}{n-1} \frac{1}{\pi^{2}+\{C+\log (n+1)\}^{2}}\left(1+\frac{e^{-C}}{n+1}\right)\right. \\
& \left.-\frac{n+1}{2^{n-1} n \pi^{2}}\right]-1-\frac{1}{k \pi^{2}} .
\end{aligned}
$$

To prove that, for certain $n$ and even $k \leqq n / 2$, coefficients $A_{k}$ are negative, it suffices to verify the inequality

$$
\begin{aligned}
C_{n}{ }^{k}\left(\frac{1}{k}+\frac{(-1)^{n}}{n-k}\right) & {\left[\frac{n-3}{n-1} \frac{1}{\pi^{2}+\{C+\log (n+1)\}^{2}}\left(1+\frac{e^{-C}}{n+1}\right)\right.} \\
& \left.-\frac{n+1}{2^{n-1} n \pi^{2}}\right]-1-\frac{1}{k \pi^{2}}>0 .
\end{aligned}
$$

5. If $n$ is even the inequality (5) will be verified for all even values of $k$ if it is verified for $k=2$; also, being verified for some even $n$, it will be verified for greater values of $n$. Now, taking $n=12$ and $k=2$, inequality (5) is verified; hence for an even $n \geqq 12, A_{k}$ with even subscripts $\leqq n / 2$ are all negative. The same being true for $n=8$ and $n=10$ we may be assured that 


$$
(-1)^{k-1} A_{k}>0, \quad 1 \leqq k \leqq \frac{n}{2},
$$

for all even $n \geqq 8$. If $n$ is odd $\geqq 13$ it suffices again to verify inequality (5) for $n=13, k=2$; by direct computation we find that it is satisfied. Hence

$$
(-1)^{k-1} A_{k}>0, \quad 1 \leqq k \leqq \frac{n-1}{2},
$$

for all odd $n \geqq 13$. It remains to be seen whether this is true for $n=11$. But by direct computation I have found the following values of Cotes coefficients for $n=11$ :

$$
\begin{aligned}
& A_{0}=A_{11}=\frac{2171465}{24 \times 10 !} ; \quad A_{1}=A_{10}=\frac{13486539}{24 \times 10 !} ; \quad A_{2}=A_{9}=-\frac{3237113}{24 \times 10 !} ; \\
& A_{3}=A_{8}=\frac{25226685}{24 \times 10 !} ; \quad A_{4}=A_{7}=-\frac{9595542}{24 \times 10 !} ; \quad A_{5}=A_{6}=\frac{15493566}{24 \times 10 !}
\end{aligned}
$$

Hence the above property of Cotes coefficients holds for all odd $n \geqq 11$, but it no longer holds for $n=9$. It remains to show that $A_{0}$ is always positive. The sign of $A_{0}$ is the same as the sign of the integral

which, by setting

$$
I_{n}=\int_{0}^{n}(1-x)(2-x) \cdots(n-x) d x,
$$

$$
\Phi(x)=\int_{0}^{x} t(1-t)(2-t) \cdots(n-t) d t
$$

can be presented thus:

$$
I_{n}=\frac{\Phi(n)}{n}+\int_{0}^{n} \frac{\Phi(x)}{x^{2}} d x .
$$

But it is known that $\Phi(x)>0$ in the interval $0<x<n$, hence $I_{n}>0$ and $A_{0}>0$.

6. For $n \equiv 3(\bmod 4)$ it is important to show that

$$
\sum_{k=0}^{(n-1) / 2} k A_{k}>\frac{n}{8}
$$

for $n \geqq 11$. To present the left hand member in a convenient form we may use formula (3). Thus we get

$$
\sum_{k=0}^{(n-1) / 2} k A_{k}=\frac{n^{2}-1}{8 n}+\frac{n+1}{n} \int_{0}^{1} \int_{0}^{1} \frac{(1-\xi)^{n} \phi(t)}{\pi^{2}+\left(\log \frac{1-\xi}{\xi} t\right)^{2}} d \xi d t
$$


where

$$
\phi(t)=\sum_{k=0}^{(n-1) / 2}(-1)^{k-1} C_{n^{k}} k\left(t^{k-1}-t^{n-k-1}\right) .
$$

By an elementary transformation this expression becomes

$$
\phi(t)=n(1-t) \psi(t),
$$

$\psi(t)$ denoting a reciprocal polynomial

$$
\begin{aligned}
& \psi(t)=1-C_{n-2}^{1} t+\cdots-C_{n-2}^{(n-5) / 2} t^{(n-5) / 2} \\
& \quad+C_{n-2}^{(n-3) / 2} t^{(n-3) / 2}-C_{n-2}^{(n-5) / 2} t^{(n-1) / 2}+\cdots+t^{n-3} .
\end{aligned}
$$

Using the following known identity,

$$
\begin{array}{r}
t^{n-2}-C_{n-2}^{1} t^{n-3}+\cdots-C_{n-2}^{(n-5) / 2} t^{(n+1) / 2} \\
=-\frac{n-3}{2} C_{n-2}^{(n-3) / 2}(1-t)^{n-2} \int_{0}^{t} \frac{y^{(n-1) / 2} d y}{(1-y)^{n-1}},
\end{array}
$$

$\psi(t)$ can be presented thus:

$$
\begin{aligned}
\psi(t)= & -\frac{n-3}{2} C_{n-2}^{(n-3) / 2}(1-t)^{n-2}\left(1+t^{-1}\right) \int_{0}^{t} \frac{y^{(n-1) / 2} d y}{(1-y)^{n-1}} \\
& +(1-t)^{n-2}+C_{n-2}^{(n-3) / 2} t^{(n-1) / 2}
\end{aligned}
$$

whence

$$
\frac{d}{d t} \frac{t \psi(t)}{(1+t)(1-t)^{n-2}}=\frac{2 t^{(n-1) / 2}}{(1-t)^{n-1}(1+t)^{2}} C_{n-2}^{(n-3) / 2}+\frac{1}{(1+t)^{2}}>0 ; t>0 .
$$

It follows that $\psi(t)>0$ for all real values of $t$ and the same is true of $\phi(t)$ if $t<1$.

This analysis shows incidentally that the algebraic equation

$$
\begin{aligned}
1-C_{n-2}^{1} t+\cdots-C_{n-2}^{(n-5) / 2} t^{(n-5) / 2}+C_{n-2}^{(n-3) / 2} & t^{(n-3) / 2} \\
& \quad-C_{n-2}^{(n-5) / 2} t^{(n-1) / 2}+\cdots+t^{n-3}=0
\end{aligned}
$$

has only imaginary roots if $n \equiv 3(\bmod 4)$; but if $n \equiv 1(\bmod 4)$ it has exactly two real roots as can be shown by similar considerations. Since

$$
\begin{aligned}
\phi(t)=n(1-t)^{n-1}+n C_{n-2}^{(n-3) / 2} t^{(n-1) / 2}(1-t) & \\
& \quad-\frac{n(n-3)}{2} C_{n-2}^{(n-3) / 2}(1-t)^{n-1}\left(1+t^{-1}\right) \int_{0}^{t} \frac{y^{(n-1) / 2} d y}{(1-y)^{n-1}}
\end{aligned}
$$


remains positive in the interval $0<t<1$ it follows that

$$
\begin{array}{r}
\sum_{k=0}^{(n-1) / 2} k A_{k}>\frac{n^{2}-1}{8 n}+\frac{n+1}{n} \int_{0}^{1 / 2}(1-\xi)^{n} G(\xi) d \xi ; \\
G(\xi)=\int_{\xi /(1-\xi)}^{1} \frac{\phi(t)}{\pi^{2}+\left(\log \frac{1-\xi}{\xi} t\right)^{2}} d t .
\end{array}
$$

But integrating by parts we find

(7)

$$
\int_{\xi /(1-\xi)}^{1} \frac{\phi(t) d t}{\pi^{2}+\left(\log \frac{1-\xi}{\xi} t\right)^{2}}>\frac{\Phi(1)}{\pi^{2}+\left(\log \frac{1-\xi}{\xi}\right)^{2}}-\frac{\Phi\left(\frac{\xi}{1-\xi}\right)}{\pi^{2}}
$$

where

$$
\Phi(x)=\int_{0}^{x} \phi(t) d t .
$$

But since

$$
\begin{aligned}
\phi(t)>n(1-t)^{n-1}+ & n C_{n-2}^{(n-3) / 2} t^{(n-1) / 2}(1-t) \\
& -\frac{n(n-3)}{2} C_{n-2}^{(n-3) / 2}(1-t)^{n-1} \int_{0}^{t} \frac{y^{(n-1) / 2}+y^{(n-3) / 2}}{(1-y)^{n-1}} d y,
\end{aligned}
$$

we find, after performing simple integrations,

$$
\Phi(1)>1+\frac{4 C_{n-2}^{(n-3) / 2}}{(n+1)(n-1)} .
$$

On the other hand

$$
\begin{aligned}
\phi(t)<n C_{n-2}^{(n-3) / 2}\left(1-\frac{n-3}{n-2}\right) & t^{(n-1) / 2}(1-t) \\
& +n(1-t)^{n-1}+\frac{n(n-1)}{2(n-2)} C_{n-2}^{(n-3) / 2} t^{(n-3) / 2}(1-t)^{2},
\end{aligned}
$$

whence

$$
\begin{aligned}
\Phi(t)<\frac{2 n C_{n-2}^{(n-3) / 2}}{(n+1)(n-2)} t^{(n+1) / 2}+1-(1-t)^{n} \\
+\frac{n(n-1)}{n-2} C_{n-2}^{(n-3) / 2}\left\{\frac{t^{(n-1) / 2}}{n-1}-\frac{2 t^{(n+1) / 2}}{n+1}+\frac{t^{(n+3) / 2}}{n+3}\right\},
\end{aligned}
$$

and after simple calculations, 


$$
\int_{0}^{1 / 2} \Phi\left(\frac{\xi}{1-\xi}\right)(1-\xi)^{n} d \xi<\frac{1}{2(n-2)}
$$

Inequalities (6), (7), (8), (9) combined give

$$
\begin{aligned}
\sum_{k=0}^{(n-1) / 2} k A_{k}-\frac{n}{8}> & {\left[\frac{n+1}{n}+\frac{4 C_{n-2}^{(n-3) / 2}}{n(n-1)}\right] \int_{0}^{1 / 2} \frac{(1-\xi)^{n} d \xi}{\pi^{2}+\left(\log \frac{1-\xi}{\xi}\right)^{2}} } \\
& -\frac{1}{8 n}-\frac{n+1}{2 \pi^{2} n(n-2)} .
\end{aligned}
$$

Now using the lower limit of the integral

$$
\int_{0}^{1 / 2} \frac{(1-\xi)^{n} d \xi}{\pi^{2}+\left(\log \frac{1-\xi}{\xi}\right)^{2}}
$$

given in $\$ 5$ we find that the right hand side of the inequality (10) is positive for $n=15$, and so

$$
\sum_{k=0}^{(n-1) / 2} k A_{k}>\frac{n}{8}
$$

for $n \geqq 15$. But this statement is true already for $n=11$ as can be ascertained directly by using values of Cotes coefficients for $n=11$ given in $\S 5$.

7. To derive the expansion of the remainder $R_{n}$ in the Newton-Cotes formula we take for starting point the well known identity valid for $0 \leqq \theta \leqq 1$

$$
\begin{array}{r}
f(\theta)=\int_{0}^{1} f(x) d x+\sum_{s=1}^{l} \frac{B_{s}(\theta)}{s !}\left\{f^{(s-1)}(1)-f^{(s-1)}(0)\right\} \\
-\int_{0}^{1} \frac{\bar{B}_{l}(\theta-t)}{l !} f^{(l)}(t) d t
\end{array}
$$

in which $B_{n}(x)$ denotes the Bernoullian polynomial of order $n$ (defined as in Nörlund's Differenzenrechnung) and $\bar{B}_{n}(x)$ is a periodic function:

$$
\begin{aligned}
\bar{B}_{n}(x) & =B_{n}(x) \text { for } 0 \leqq x<1, \\
\bar{B}_{n}(x+1) & =\bar{B}_{n}(x) \text { for all } x .
\end{aligned}
$$

Take $l=2 \nu=n+1$ or $n+2$ according as $n$ is odd or even. Set $\theta=0,1 / n$, $2 / n, \cdots, 1$, multiply the resulting equations by the corresponding Cotes coefficients and take the sum; the result will be 


$$
\begin{aligned}
\sum_{i=0}^{n} A_{i} f\left(\frac{i}{n}\right)= & \int_{0}^{1} f(x) d x+\frac{\sum_{i=0}^{n} A_{i} B_{2 v}\left(\frac{i}{n}\right)}{(2 \nu) !}\left\{f^{(2 \nu-1)}(1)-f^{(2 \nu-1)}(0)\right\} \\
& -\int_{0}^{1} \frac{f^{(2 \nu)}(t)}{(2 \nu) !} \sum_{i=0}^{n} A_{i} \bar{B}_{2 v}\left(\frac{i}{n}-t\right) d t
\end{aligned}
$$

since for $s=1,2, \cdots, 2 \nu-1$

$$
\sum_{i=0}^{n} A_{i} B_{8}\left(\frac{i}{n}\right)=\int_{0}^{1} B_{8}(x) d x=0 .
$$

Setting for brevity

$$
\sum_{i=0}^{n} A_{i}\left\{\bar{B}_{p}\left(\frac{i}{n}-t\right)-B_{p}\left(\frac{i}{n}\right)\right\}=G_{p}(t)
$$

it follows from (11) that

$$
R_{n}=\frac{1}{(2 \nu) !} \int_{0}^{1} G_{2 v}(t) f^{(2 v)}(t) d t .
$$

From the definition of $G_{p}(t)$ we see that

$$
G_{p}(0)=G_{p}(1)=0,
$$

and without any difficulty (owing to the fact that $A_{n-i}=A_{i}$ ) we can establish the following relations:

$$
G_{p}(1-t)=(-1)^{p} G_{p}(t), \sum_{i=0}^{n} A_{i} B_{28-1}\left(\frac{i}{n}\right)=0,
$$

so that $G_{2 s-1}(t)$ can be written simply thus:

$$
G_{2 s-1}(t)=\sum_{i=0}^{n} A_{i} \bar{B}_{2 s-1}\left(\frac{i}{n}-t\right),
$$

and from this expression, because

$$
\bar{B}_{n}^{\prime}(x)=n \bar{B}_{n-1}(x),
$$

the following relations can be derived:

$$
\begin{aligned}
G_{2 s}^{\prime}(t) & =-2 s G_{2 s-1}(t) \\
G_{2 s+1}^{\prime \prime}(t) & =2 s(2 s+1) G_{2 s-1}(t), \\
G_{2 s}^{\prime \prime}(t) & =2 s(2 s-1)\left[G_{2 s-2}(t)+\sum_{i=0}^{n} A_{i} B_{2 s-2}\left(\frac{i}{n}\right)\right] .
\end{aligned}
$$


8. For $s \geqq \nu$ functions $G_{2 s}(t)$ do not change sign in the interval $0<t<1$. To prove this fundamental property, let $\beta_{s}$ and $\alpha_{s}$ represent, respectively, the number of times $G_{2 s}(t)$ and $G_{2 s-1}(t)$ change their sign when $t$ increases from 0 to 1 . Because $G_{2 s}(0)=G_{2 s}(1)=0$ it follows from (13) and Rolle's theorem that

$$
\beta_{s}+1 \leqq \alpha_{s} .
$$

Again, using (14) and applying Rolle's theorem twice, we get

$$
\alpha_{s} \leqq \alpha_{s-1},
$$

so that for $s \geqq \nu$,

Since

$$
\beta_{s}+1 \leqq \alpha_{v}
$$

$$
G_{2 p-1}(1-t)=-G_{2 \nu-1}(t),
$$

it follows that $\alpha_{\nu} \geqq 1$ and it is important to prove that $\alpha_{\nu}=1$.

To this end we notice first that for $0 \leqq t \leqq 1$

$$
\begin{aligned}
G_{2 v-1}(t) & =\sum_{i=0}^{n} A_{i} \bar{B}_{2 v-1}\left(\frac{i}{n}-t\right) \\
& =\sum_{i=0}^{n} A_{i} B_{2 v-1}\left(\frac{i}{n}-t\right)+(2 \nu-1) \sum_{i \leqq n t} A_{i}\left(\frac{i}{n}-t\right)^{2 \nu-2} .
\end{aligned}
$$

Furthermore, $B_{2 v-1}(x)$ being a polynomial of degree $2 \nu-1$,

$$
\begin{aligned}
\sum_{i=0}^{n} A_{i} B_{2 \nu-1}\left(\frac{i}{n}-t\right) & =\int_{0}^{1} B_{2 \nu-1}(x-t) d x \\
& =\frac{1}{2 \nu}\left\{B_{2 \nu}(1-t)-B_{2 \nu}(-t)\right\}=-t^{2 \nu-1} .
\end{aligned}
$$

Hence $G_{2 v-1}(t)$ differs only by a constant factor from the function

$$
R_{0}(t)=\frac{t^{2 \nu-1}}{2 \nu-1}-\sum_{i \leqq n t} A_{i}\left(\frac{i}{n}-t\right)^{2 v-2},
$$

and the number of times this function changes sign in the interval $0<t<1$ is $\alpha_{\nu}$.

We shall now prove the following Fundamental Lemma:

Fundamental Lemma. The function $R_{0}(t)$ changes sign once and only once in the interval $0<t<1$, so that $\alpha_{\nu}=1$.

Let 


$$
R_{k}(t)=\frac{(-1)^{k} t^{2 \nu-k-1}}{2 \nu-k-1}-\sum_{i \leqq n t} A_{i}\left(\frac{i}{n}-t\right)^{2 \nu-k-2}
$$

for $k=0,1,2, \cdots, 2 \nu-2$. These functions differ only by constant factors from the successive derivatives of $R_{0}(t)$. With the exception of $R_{2 v-2}(t)$ they are continuous, but

$$
R_{2 \nu-2}(t)=t-\sum_{i \leqq n t} A_{i}
$$

has discontinuities at the points $1 / n, 2 / n, \cdots,(n-1) / n$. By the fundamental property of the Newton-Cotes formula,

$$
R_{k}(1)=R_{k}(0)=0 .
$$

Also

$$
R_{k}(1-t)=(-1)^{k-1} R_{k}(t),
$$

whence it follows that for an even subscript $k$ there is always a change of sign at $t=\frac{1}{2}$.

Let $N_{k}$ in general represent the number of changes of sign of $R_{k}(t)$ in the interval $0<t<1$. Then by Rolle's theorem

$$
N_{k}+1 \leqq N_{k+1}
$$

for $k=0,1,2, \cdots, 2 \nu-3$. Hence

$$
N_{0}+2 \nu-2 \leqq N_{2 v-2}
$$

and also

$$
N_{0}+2 \nu-3 \leqq N_{2 \nu-3} .
$$

Now it is possible to assign a certain upper limit to $N_{2 v-2}$. To this end we distinguish the following cases:

Case 1: $n=2 \nu-2 \geqq 8$ and divisible by 4 . The interval $0<t<\frac{1}{2}$ may be divided into $(\nu+1) / 2$ intervals:

$$
\begin{aligned}
0<t<\frac{1}{n} ; \frac{1}{n}<t< & \frac{3}{n} ; \frac{3}{n}<t<\frac{5}{n} ; \cdots ; \frac{\nu-4}{n}<t<\frac{\nu-2}{n} ; \\
& \frac{\nu-2}{n}<t<\frac{\nu-1}{n} .
\end{aligned}
$$

In the interval $0<t<1 / n$, since $A_{0}>0, R_{2 v-2}(t)$ might change sign, but not more than once. In the last interval $(\nu-2) / n<t<(\nu-1) / n$ no changes of sign occur, since $R_{2 v-2}(t)$ changes its sign at $t=\frac{1}{2}$ and $A_{\nu-1}<0$. In each of the intervals of the type $(2 k-1) / n<t<(2 k+1) / n$ the sign may change at most 
once, since $A_{2 k}<0$. Considering that $R_{2 \nu-2}(t)$ may change sign at end points of the intervals, the total number of changes of sign in the interval $0<t<\frac{1}{2}$ cannot exceed $\nu-1$; in the interval $\frac{1}{2}<t<1$ there is exactly the same number of changes of sign and the sign changes at $t=\frac{1}{2}$. Hence $N_{2 \nu-2} \leqq 2 \nu-1$ and $N_{0}+2 \nu-2 \leqq N_{2 \nu-2}$ and this necessarily implies $N_{0}=1, N_{2 \nu-2}=2 \nu-1$.

Case $2: n=2 \nu-2 \geqq 10$ and $n \equiv 2(\bmod 4)$. By very similar considerations we find again $N_{2 \nu-2} \leqq 2 \nu-1$, whence $N_{0}=1, N_{2 \nu-2}=2 \nu-1$.

Case 3: $n=2 \nu-1 \geqq 13, \nu$ odd. Divide again the interval $0<t<\frac{1}{2}$ into $(\nu+3) / 2$ parts:

$$
\begin{aligned}
0<t<\frac{1}{n} ; \frac{1}{n}<t<\frac{3}{n} ; & \cdots ; \frac{\nu-4}{n}<t<\frac{\nu-2}{n} ; \frac{\nu-2}{n}<t<\frac{\nu-1}{n} \\
& \frac{\nu-1}{n}<t<\frac{1}{2} .
\end{aligned}
$$

In the first interval there might be at most one change of sign. There are no changes of sign in the last interval, since $R_{2 \nu-2}(t)$ passes at $t=\frac{1}{2}$ from negative to positive values. Neither does the sign change in the interval $(\nu-2) / n<t$ $<(\nu-1) / n$, since at the right end of this interval $R_{2 v-2}(t)$ is negative. Altogether $R_{2 \nu-2}(t)$ in the interval $0<t<\frac{1}{2}$ cannot have more than $(\nu-1) / 2$ $+(\nu-1) / 2=\nu-1$ changes of sign. Hence $N_{2 \nu-2} \leqq 2 \nu-1$ and again $N_{0}=1$, $N_{2 \nu-2}=2 \nu-1$.

Case 4: $n=2 \nu-1 \geqq 11, \nu$ even. In a similar manner we find $2 \nu+1$ as the upper limit of $N_{2 v-2}$. The inequality $N_{0}+2 \nu-2 \leqq 2 \nu+1$ shows only that either $N_{0}=1$ or $N_{0}=3$. To remove the last possibility we take into consideration $R_{2 \nu-3}(t)$. First from the inequality $N_{2 \nu-3}+1 \leqq N_{2 \nu-2}$ we conclude $N_{2 \nu-3} \leqq 2 \nu$. Further, for small values of $t$

$$
R_{2 \nu-3}(t)=-\frac{t^{2}}{2}+A_{0} t>0
$$

and

$$
R_{2 v-3}\left(\frac{1}{2}\right)=-\frac{1}{8}+\frac{1}{2} \sum_{i=0}^{(n-1) / 2} A_{i}-\frac{1}{n} \sum_{i=0}^{(n-1) / 2} i A_{i}=\frac{1}{8}-\frac{\sum_{i=0}^{(n-1) / 2} i A_{i}}{n}<0
$$

because

$$
\sum_{i=0}^{(n-1) / 2} i A_{i}>\frac{n}{8}
$$

in case $n \equiv 3(\bmod 4)$ and $n \geqq 11$. Thus $\frac{1}{2} N_{2 v-3}$ is an odd number, hence $N_{2 p-3} \leqq 2 \nu-2$. From the inequality 


$$
N_{0}+2 \nu-3 \leqq N_{2 \nu-3} \leqq 2 \nu-2
$$

we conclude again $N_{0}=1, N_{2 \nu-3}=2 \nu-2$. This proof does not apply to $n=2$, $4,6,3,5,7,9$. But the truth of the Lemma in these particular cases can be verified directly. This verification is not very laborious, since everything reduces to testing whether certain algebraic equations have roots between 0 and 1 , and in all cases this is easily decided by application of the well known and simple Laguerre criterion.

9. Now that the equality $\alpha_{\nu}=1$ is proved it follows immediately that $\beta_{s}=0$ for $s \geqq \nu$, that is, $G_{2 s}(t)$ does not change sign in the interval $0<t<1$. It suffices now to make use of formula (15) and apply repeatedly the integration by parts in (12) to obtain the following expansion of $R_{n}$ :

$$
R_{n}=\sum_{s=0}^{k-1} c_{s}\left\{f^{(2 \nu+2 s-1)}(1)-f^{(2 \nu+2 s-1)}(0)\right\}+c_{k} f^{(2 v+2 k)}(\xi),
$$

where

$$
c_{s}=-\frac{1}{(2 \nu+2 s) !} \sum_{i=0}^{n} A_{i} B_{2 v+2 s}\left(\frac{i}{n}\right)=-\frac{\gamma_{2 \nu+2 s}}{(2 \nu+2 s) !}
$$

and $\xi$ is an unknown number between 0 and 1 . To show that this expansion possesses all the properties of the Euler-Maclaurin expansion it remains to prove that the numbers

$$
\gamma_{2 v}, \gamma_{2 v+2}, \gamma_{2 v+4}, \cdots
$$

alternate in sign. But this is almost evident if we notice that

$$
\int_{0}^{1} G_{2 v+2 s}(t) d t=-\gamma_{2 v+2 s}
$$

and that $G_{2 v+28}(t)$ has the same sign as

$$
G_{2 \nu+2 s}^{\prime \prime}(0)=(2 \nu+2 s)(2 \nu+2 s-1) \gamma_{2 \nu+2 s-2} .
$$

It is easy to see that $c_{0}$ is negative, so that in general

$$
(-1)^{s-1} c_{s}>0 \text {. }
$$

The expansion (16) is especially useful when all the derivatives of an even order $\geqq 2 \nu$ have the same sign in $(0,1)$. For then, retaining a certain number of terms in (16), the error in estimating $R_{n}$ will be less in absolute value than the first neglected term and of the same sign.

10. Perhaps the simplest way of calculating coefficients $c_{s}$ is to use their 
expression (17). Since values of $B_{28}(x)$ can be expressed simply through Bernoullian numbers for $x=\frac{1}{2}, \frac{1}{3}, \frac{1}{4}, \frac{1}{6}$, we have for $n=2,3,4,6$ very elegant expansions:

$$
\begin{aligned}
\int_{0}^{1} f(x) d x= & \frac{1}{6}\left\{f(0)+4 f\left(\frac{1}{2}\right)+f(1)\right\} \\
& +\sum_{k=2,3, \cdots} \frac{(-1)^{k-1}\left(1-2^{2-2 k}\right)}{3} \frac{B_{k}}{(2 k) !}\left\{f^{(2 k-1)}(1)-f^{(2 k-1)}(0)\right\}, \\
\int_{0}^{1} f(x) d x= & \frac{1}{8}\left\{f(0)+3 f\left(\frac{1}{3}\right)+3 f\left(\frac{2}{3}\right)+f(1)\right\} \\
& +\sum_{k=2,3, \cdots} \frac{(-1)^{k-1}\left(1-3^{2-2 k}\right)}{8} \frac{B_{k}}{(2 k) !}\left\{f^{(2 k-1)}(1)-f^{(2 k-1)}(0)\right\}, \\
\int_{0}^{1} f(x) d x= & \frac{1}{90}\left\{7 f(0)+32 f\left(\frac{1}{4}\right)+12 f\left(\frac{1}{2}\right)+32 f\left(\frac{3}{4}\right)+7 f(1)\right\} \\
+\sum_{k=3,4, \cdots}(-1)^{k}-5 \cdot 2^{2-2 k}+4 \cdot 4^{2-2 k} & \frac{B_{k}}{(2 k) !}\left\{f^{(2 k-1)}(1)-f^{(2 k-1)}(0)\right\}, \\
\int_{0}^{1} f(x) d x= & \frac{1}{840}\left\{41 f(0)+216 f\left(\frac{1}{6}\right)+27 f\left(\frac{1}{3}\right)+272 f\left(\frac{1}{2}\right)\right. \\
& \left.+27 f\left(\frac{2}{3}\right)+216 f\left(\frac{5}{6}\right)+41 f(1)\right\} \\
& +\sum_{k=4,5, \cdots}(-1)^{k-1} \frac{1-7 \cdot 2^{4-2 k}+7 \cdot 3^{4-2 k}-6^{4-2 k}}{840} \frac{B_{k}}{(2 k) !} \times\left\{f^{(2 k-1)}(1)-f^{(2 k-1)}(0)\right\} .
\end{aligned}
$$

The following table gives values of $\gamma_{2 v}, \gamma_{2 v+2}, \gamma_{2 v+4}, \gamma_{2 v+6}$ for $n=5,7,8$ :

\begin{tabular}{|c|cccc|}
\hline$n$ & $\gamma_{2 v}$ & $\gamma_{2 \nu+2}$ & $\gamma_{2 \nu+4}$ & $\gamma_{2 \nu+6}$ \\
\hline 5 & $\frac{11}{2^{2} \cdot 3 \cdot 7 \cdot 5^{4}}$ & $-\frac{7}{2 \cdot 3 \cdot 5^{5}}$ & $\frac{15351}{2^{2} \cdot 11 \cdot 5^{8}}$ & $-\frac{64427}{3 \cdot 7 \cdot 13 \cdot 5^{7}}$ \\
\hline 7 & $\frac{167}{2 \cdot 3^{2} \cdot 5 \cdot 7^{6}}$ & $-\frac{2665}{2 \cdot 3 \cdot 11 \cdot 7^{7}}$ & $\frac{1387331}{3 \cdot 5 \cdot 13 \cdot 7^{9}}$ & $-\frac{103112581}{3^{2} \cdot 7^{12}}$ \\
\hline 8 & $\frac{37}{2^{19} \cdot 3 \cdot 11}$ & $-\frac{235873}{2^{24} \cdot 3 \cdot 5 \cdot 7 \cdot 13}$ & $\frac{134671787}{2^{31} \cdot 3^{2} \cdot 5}$ & $-\frac{180237358327}{2^{32} \cdot 3^{2} \cdot 5 \cdot 17}$ \\
\hline
\end{tabular}


It might be of interest for practical computers to know that the remainder in the much used G. F. Hardy's formula can be expanded in the same manner. We have in fact

$$
\begin{aligned}
& \int_{0}^{1} f(x) d x=\frac{1}{3}\left[0.14 f(0)+0.81 f\left(\frac{1}{6}\right)+1.1 f\left(\frac{1}{2}\right)+0.81 f\left(\frac{5}{6}\right)+0.14 f(1)\right] \\
& +\frac{1}{21772800}\left\{f^{(5)}(1)-f^{(5)}(0)\right\} \\
& +\sum_{k=4,5,6}(-1)^{k-1} \frac{1-29 \cdot 2^{1-2 k}+81 \cdot 3^{1-2 k}-81 \cdot 6^{1-2 k}}{300} \\
& \times \frac{B_{k}}{(2 k) !}\left\{f^{(2 k-1)}(1)-f^{(2 k-1)}(0)\right\} .
\end{aligned}
$$

Stanford University, CaltF. 